AperTO - Archivio Istituzionale Open Access dell'Università di Torino

\title{
An unusual calf lesion in an immunocompromised patient.
}

\section{This is the author's manuscript}

Original Citation:

Availability:

This version is available http://hdl.handle.net/2318/1668535

since 2018-05-20T18:04:35Z

Published version:

DOI:10.1007/s11739-018-1878-y

Terms of use:

Open Access

Anyone can freely access the full text of works made available as "Open Access". Works made available under a Creative Commons license can be used according to the terms and conditions of said license. Use of all other works requires consent of the right holder (author or publisher) if not exempted from copyright protection by the applicable law. 
Gili M.*, Chiacchiarini F.*, Morra di Cella S.*, Baglioni E.**, Brizzi MF.*, De Rosa F.G.*, Porta $M^{*}$.

\section{AN UNUSUAL CALF LESION IN AN IMMUNOCOMPROMISED PATIENT}

*Department of Medical Sciences, University of Turin,

** Department of General and Specialized Surgery, Città della Salute e della Scienza di Torino

\section{Corresponding author:}

Prof. Massimo Porta, MD PhD

massimo.porta@unito.it

Tel. +390116336028

ORCID: 0000-0002-3407-6017 


\section{Prof. Porta}

Dear colleagues, we present today a rare infection developed in an immunocompromised patient, which represented a diagnostic challenge while admitted to our Internal Medicine ward. Dr. Gili, will you please describe the patient?

\section{Case presentation}

\section{Dr. Gili}

A 71 year old man affected by chronic lymphatic leukemia, arrived in the emergency department with a swollen and tender right calf. The patient reported a $7 \mathrm{Kg}$ weight loss over the previous month and his current therapy included antiviral (Acyclovir $400 \mathrm{mg}$ bid) and trimethoprimsulfamethoxazole (160 mg twice weekly) prophylaxis, prednisone $25 \mathrm{mg} / \mathrm{die}$ and a proton pump inhibitor.

Clinical examination showed normal vital parameters except for mild fever and confirmed swelling, approximately $8 \mathrm{~cm}$ in length, of the postero-medial region of right calf. Laboratory findings revealed mild anemia, elevated $\mathrm{C}$ reactive protein levels and procalcitonin within the normal range. A previously known lesion of the left lung appeared enlarged at chest X-Ray, and soft tissue echography interpreted the calf swelling as a large organizing hematoma of the medial gastrocnemius muscle. Antibiotic therapy with amoxicillin-clavulanate $2,2 \mathrm{gr}$ iv tid and azithromycin $500 \mathrm{mg}$ od was started, as superinfection of the hematoma and/or the pulmonary lesion was hypothesized, and the patient was admitted to our ward.

A diagnosis of chronic lymphatic leukemia had been established in 2006 and the patient had received six cycles of chemo- and immunotherapy, comprehensive of some experimental protocols, but these had not been able to stop progression of the disease. One year before the current admission, following administration of the experimental drug idelalisib, a re-staging chest computed tomography (CT) had revealed a nodule, one centimeter in diameter, at the left pulmonary apex (Fig.1a) which the radiologists were unable to further characterize. Considering its relatively rapid development, the most plausible hypotheses were either a new neoplastic lesion or an intra-pulmonary lymph node. Among further microbiologic and imaging investigations, Quantiferon test and neoplasia markers (CEA/NSE/Cyfra21-1/cromogranina A) were indifferent, while a Positron Emission Tomography / Computed Tomography (PET/CT) scan documented intense accumulation of the radiotracer in correspondence of the nodule. To check if the lesion would respond to antimicrobial therapy, azythromicin was started on an empirical basis.

CT scans had been repeated every three months over the following year to monitor the lesion, which continued to expand, reaching $6 \mathrm{~cm}$ in main diameter in August 2015 (Fig. 1b). The 
haematologists in charge decided to interrupt treatment with Ofatutmumab and perform a bronchoscopy with broncho-alveolar lavage and trans-bronchial biopsy. Since cultural, cytological and histological examinations were all negative, a trans-parietal CT-guided biopsy was performed, which revealed an inflammatory pattern but no neoplastic cells.

Meanwhile, the patient was admitted to our ward because of the calf lesion and, to complete the pulmonary diagnostic assessment, a new chest CT scan was performed, followed by a PET scan to better direct a new lung biopsy. An irregular pulmonary consolidation extended to the third posterior costal arch and the posterior thorax wall, corresponding to the site of the previous pulmonary biopsy. The trans-parietal CT-guided lung biopsy that followed confirmed an inflammatory pattern, without any clues to the nature of the lesion. An interdisciplinary consultation (hematologist, thoracic surgeon and internists) recommended that the lung lesion be surgically removed after resolution of the calf infection.

Antibiotic therapy was continued but did not improve swelling and inflammation of the calf. A plastic surgeon was consulted.

\section{Dr. Baglioni}

The lesion had the appearance of a large, possibly superinfected, muscle hematoma but did not respond to usual therapies, including compression stockings, bed rest, lifting of the leg, or antimicrobial therapy. I drained the collection, obtaining abundant serum-hematic fluid and left a drainage on site (Fig. 2a-b-c). Fluid accumulation relapsed upon removal of the drainage, so that surgical debridement of the calf abscess was performed and the resulting open wound treated by negative pressure therapy, (V.A.C. therapy, KCI- Kci Medical Srl) until hospital discharge.

\section{Dr. Gili:}

While waiting for results of the fluid culture, the patient complained of worsening pain at the calf and fever at night. Samples for blood cultures were collected, while X-Ray and magnetic resonance ruled out osteomyelitis of the right calf. After 5 days, blood cultures did not grow any pathogens. A clinical suspicion of Nocardia infection was raised by the presence of macroscopic inclusions, resembling fungal hyphae, in the abscess fluid. Nocardia bacteria aggregate in filaments similar to hyphae and are common opportunistic pathogens in immunodepressed patients. Therefore, the clinical presentation, the negative blood cultures and procalcitonin within the normal range [1], strengthened our suspicion and the microbiologists were alerted. Empirical antimicrobial treatment was continued until, after another 2 days, cultures identified the presence of Nocardia in the abscess fluid. An infective disease consultant was brought in. 


\section{Prof. De Rosa}

Nocardiosis is a granulomatous pyogenic infection caused by species belonging to the aerobic Actinomycetes group. Nocardia is a non-spore forming, gram-positive filamentous bacillus with hyphae-like branching. It is saprophytic and can be found in soil and water [2,3]. The main risk factor for infection is a defective immune system, consequent to diabetes, malignancies, HIV/AIDS, lung disorders, connective tissue disorders, alcoholism, transplant, and steroid therapy [4-6]. Species associated with human infection include the Nocardia asteroides complex (more than 50\% of cases in humans), $N$. brasiliensis, $N$. abscessus, $N$. cyriacigeorgica, $N$. farcinica, $N$. nova and others. $[2,4,5]$. These bacteria are universally spread, being isolated worldwide in individuals of any age and equally in both sexes [4,5]. Differentiation of Nocardia species is not routinely performed in most laboratories, because the procedure is expensive and not expected to modify treatment.

\section{Prof. Porta}

How is the microbiological workout performed in the suspicion of nocardiosis?

\section{Prof. De Rosa}

Nocardia can be identified in biopsies, sputum, pus or CSF. Culture is performed with the BCYE medium (buffered agar with yeast and charcoal extracts) and the branching pseudo-hyphae are identified by Gram staining. Culture should be prolonged for at least two weeks. The forming colonies create rosary aggregates of Gram+ Nocardiae showing weak acid-resistance [2,3,7]. Differential diagnosis should consider other slow-developing pulmonary diseases such as fungal infections, aspergillosis, mucormycosis, histoplasmosis, blastomycosis, cryptococcosis, actinomycosis, mycobacterial infections, lung malignancies, lymphocutaneous diseases, superficial cellulitis, mycetoma, brain malignancies, abscesses or vascular infarctions [5].

\section{Prof. Porta}

How ìs nocardiosis usually manifested?

\section{Dr. Chiacchiarini}

Clinical signs depend on the infection site. Inhalation may cause pulmonary nocardiosis, pneumonia, lung abscesses or cavitary lesions, with subclinical symptoms such as fatigue, lowgrade fever, shivering, cough, dyspnea, pleuritic pain, loss of weight. [4,5,7,8] Access of the bacteria via a cut or abraded skin causes cutaneous nocardiosis, that may occur also in non- 
immunocompromised patients and is characterized by cellulitis, ulcers, pyoderma, ulcerations or abscesses. The infection can spread from the skin to loco-regional lymph nodes or subcutaneous layers [5,9]. In immunocompromised patients it frequently disseminates to non-contiguous sites such as brain, kidneys, joints, heart, eyes, and bone. Consequently, neuroimaging and bone magnetic resonance should be performed in every patient affected by nocardiosis. [4-6]

\section{Dr. Morra di Cella}

To exclude systemic dissemination of the infection, a brain magnetic resonance was performed and a swab from the inflamed skin area at the site of the previous trans-parietal lung biopsy was sent for culture. Bilateral micro-abscesses in the cortex were detected and skin localization of Nocardia was confirmed.

\section{Prof. Porta}

What is the appropriate therapy for this condition?

\section{Prof. Brizzi}

If not treated, the infection may be fatal. The majority of patients respond, albeit slowly, to first-line therapy with trimethoprim-sulphamethoxazole (TMP-SMX) (trimethoprim dose: 2,5-5 mg/kg bid per os or $15 \mathrm{mg} / \mathrm{kg}$ tid ev) [4,5]. The drug has excellent penetration into most tissue compartments, including the central nervous system, and reaches high serum concentrations even after oral administration [4]. Concern is growing following recent reports of sulfonamide resistance, especially in Nocardia farcinica [5,10,11]. Alternatives in case of resistance or allergy to sulfonamides are amikacin (7,5 mg/kg bid), imipenem (500 mg every 6 hours), ceftriaxone, and linezolid [4,12]. Duration of therapy depends on the site of infection and presence of immunodeficiency.

One to three months of oral TMP-SMX or amoxicillin-clavulanate are sufficient to treat mild cutaneous infections, extended to 6-12 months in immunocompromised patients. Combined therapy with TMP-SMX (15 mg/kg iv of trimetoprim in 2-4 doses/die) and amikacin (7.5 mg/kg iv every 12 h) is recommended in severe pulmonary or disseminated infections and in immunocompromised patients. An alternative is imipenem (500 mg iv every 6 hours) plus amikacin. If the brain is involved, TMP-SMX is the therapy of choice, with imipenem and a third generation cephalosporin (ceftriaxone 2 g every 12 hours), in association with amikacin if multi-organ infection is present. 
There are few experiences with linezolid, showing high degree of success, especially if the central nervous system is involved [14].

Surgical drainage of abscesses with careful debridement and wound drainage are indicated $[4,5,12,13]$. After $3-6$ weeks of iv treatment it is possible to shift to oral therapy, which should be continued for 6-12 months if the infection is disseminated or the brain is involved. Immunocompromised patients may also require prolonged iv therapy, up to 3-6 months [4,5,12,13].

\section{Dr. Chiacchiarini}

We reached a final diagnosis of disseminated nocardiosis involving the lung, skin, deep soft tissues and, possibly, brain micro-abscesses in an immunocompromised patient affected by chronic lymphatic leukemia on steroid therapy. Antimicrobial therapy with i.v. trimethoprimsulphamethoxazole (trimethoprim $15 \mathrm{mg} / \mathrm{kg}$ ) tid was started.

\section{Dr. Morra di Cella}

After one month of antimicrobial therapy a CT scan of the thorax revealed significant reduction in size of the lesion at the left pulmonary apex, but the lymphoproliferative pathology was rapidly progressing with significant increase in volume of supra- and infra diaphragmatic lymph nodes. The calf was improving, after surgical debridement combined with antimicrobial therapy. The patient was discharged from hospital and continued VAC therapy and oral TMP-SMX at home. His conditions remained stable for three months, but then fever and calf tenderness and swelling reappeared. The patient was re-admitted to our division and re-staging of the infection was performed. The lung lesion presented complete fibrotic involution, the lymphadenopaties were stable in appearance and the brain micro-abscesses had completely disappeared. However, inflammation and fluid collection were still present in the calf. A new surgical procedure was performed, with removal of the medial gastrocnemius muscle followed by a new course of VAC therapy.

\section{Prof. De Rosa}

The subcutaneous infection had relapsed despite continuous TMP-SMX therapy and cultures from surgical drainage were now positive for Cotrimoxazole-resistant Nocardia. My advice was to start cefepime $2 \mathrm{~g}$ tdi and amikacin 7,5 $\mathrm{mg} / \mathrm{Kg}$ bid iv for the first 15 days, to be replaced with ceftriaxone at discharge $[5,6]$. 


\section{Dr. Morra di Cella}

This last regimen administered finally eradicated the infection from the soft tissues of the calf. After two months of the new treatment the drainage was removed and local signs of inflammation had disappeared, so that antimicrobial therapy could be interrupted. Treatment for the hematological disease could now be re-started.

\section{Prof. Brizzi}

A seventh line therapy with Ibrutinib was started with excellent results. Hematopoiesis progressively improved until periodic blood transfusions were no longer necessary, lymphadenopathies regressed and the infection did not reappear.

\section{Prof. Porta}

A brief commentary is in order to sum up this case. As nocardiosis is quite rare, it is difficult to suspect and diagnose. Clinical signs are non-specific and the bacterium grows slowly and only in particular culture media. Clinicians should suspect nocardiosis in immunocompromised patients presenting with pulmonary and/or cutaneous infections. The appropriate duration of antimicrobial therapy and the threat of antibiotic resistance must also be kept in mind. Usually, patients with hematological neoplasia receive TMP-SMX prophylaxis, which is effective in the prevention of Pneumocystis pneumonia but decrease the risk of nocardial infection only if assumed daily. Intermittent regimens ( 2 double-strength tablets twice weekly or 1 single strength tablet 3 times weekly) are less protective. [5] Unfortunately, our patient had not been under TMP-SMX prophylaxis while treated with biologic agents for his leukemia.

\section{Compliance with Ethical Standards}

Conflict of Interest: The authors declare that they have no conflict of interest.

Informed consent was obtained from the patient before applying the diagnostic and therapeutic procedures described. 


\section{References:}

1. Pieralli F, Corbo L, Torrigiani A, Mannini D, Antonielli E, Mancini A, Corradi F, Arena F, Moggi Pignone A, Morettini A, Nozzoli C, Rossolini GM (2017) Usefulness of procalcitonin in differentiating Candida and bacterial blood stream infections in critically ill septic patients outside the intensive care unit. Int Emerg Med 12: 629-635.

2. McHugh KE, Sturgis CD, Procop GW, Rhoads DD. (2017) The cytopathology of Actinomyces, Nocardia, and their mimickers. Diagn Cytopathol. 45:1105-1115.

3. Sullivan DC, Chapman SW. (2010) Bacteria that masquerade as fungi: actinomycosis/nocardia. Proc Am Thorac Soc. 7:216-21.

4. Kandi V. (2015) Human Nocardia Infections: A Review of Pulmonary Nocardiosis; Cureus. 7:e304.

5. Wilson JW. (2012). Nocardiosis: updates and clinical overview. Mayo Clin Proc. 87:403-7.

6. Abreu C, Rocha-Pereira N, Sarmento A, Magro F. (2015) Nocardia infections among immunomodulated inflammatory bowel disease patients: A review. World J Gastroenterol. 21:6491-8.

7. Agterof MJ, van der Bruggen T, Tersmette M, ter Borg EJ, van den Bosch JM, Biesma DH. (2007) Nocardiosis: a case series and a mini review of clinical and microbiological features. Neth J Med. 65:199-202.

8. Kurahara Y, Tachibana K, Tsuyuguchi K, Akira M, Suzuki K, Hayashi S. (2014) Pulmonary nocardiosis: a clinical analysis of 59 cases. Respir Investig. 52:160-6.

9. Pai S, Pai K, Sharma S. (2015) Cutaneous nocardiosis: an underdiagnosed pathogenic infection. BMJ Case Rep. 2015. 
10. Valdezate S, Garrido N, Carrasco G, Villalón P, Medina-Pascual MJ, Saéz-Nieto JA. (2015) Resistance gene pool to co-trimoxazole in non-susceptible Nocardia strains. Front Microbiol. 6:376.

11. Brown-Elliott BA, Biehle J, Conville PS, Cohen S, Saubolle M, Sussland D, Wengenack N, Kriel K, Bridge L, McNulty S, Vasireddy R, Wallace RJ Jr. (2012) Sulfonamide resistance in isolates of Nocardia spp. from a US multicenter survey. J Clin Microbiol. 50:670-2.

12. De La Cruz O, Minces LR, Silveira FP. (2015) Experience with linezolid for the treatment of nocardiosis in organ transplant recipients. J Infect. 70:44-51.

13. Xu Q, Zhan R, Feng Y, Chen J. (2015) Successful treatment of multifoci nocardial brain abscesses: a case report and literature review. Medicine (Baltimore) 94:e848.

14. Moylett EH, Pacheco SE, Brown-Elliott BA, Perry TR, Buescher ES, Birmingham MC, Schentag JJ, Gimbel JF, Apodaca A, Schwartz MA, Rakita RM, Wallace RJ Jr. (2003) Clinical experience with linezolid for the treatment of nocardia infection. Clin Infect Dis. 36:313-8. 


\section{Figure legends:}

Fig.1. CT-scan evolution of the lesion of the left pulmonary apex from first detection (left) to ten months later (right).

Fig. 2. From left to right: Appearance of the fluid drained from the calf lesion; X-Ray confirming correct placement of the drainage; collection bag with the full amount of drained fluid. 
Fig.1. CT-scan evolution of the lesion of the left pulmonary apex from first detection (left) to ten months later (right).
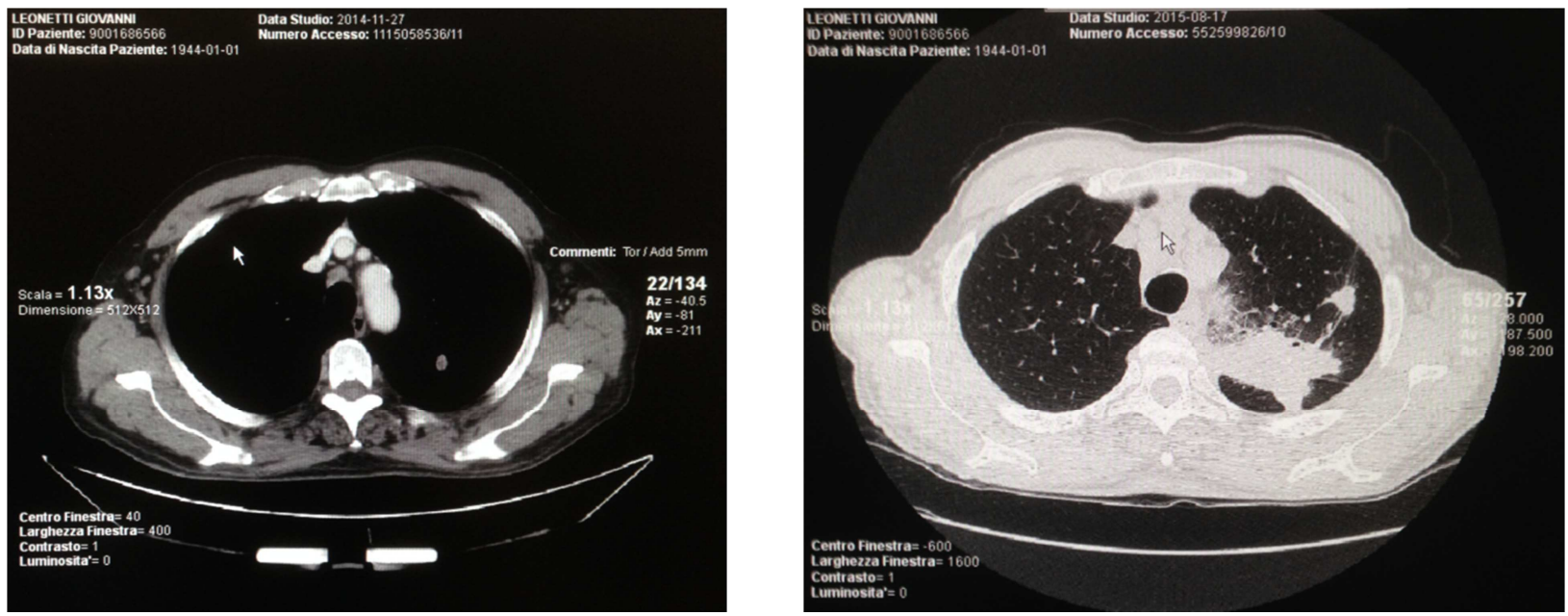
Fig. 2. From left to right: Appearance of the fluid drained from the calf lesion; X-Ray confirming correct placement of the drainage; collection bag with the full amount of drained fluid.
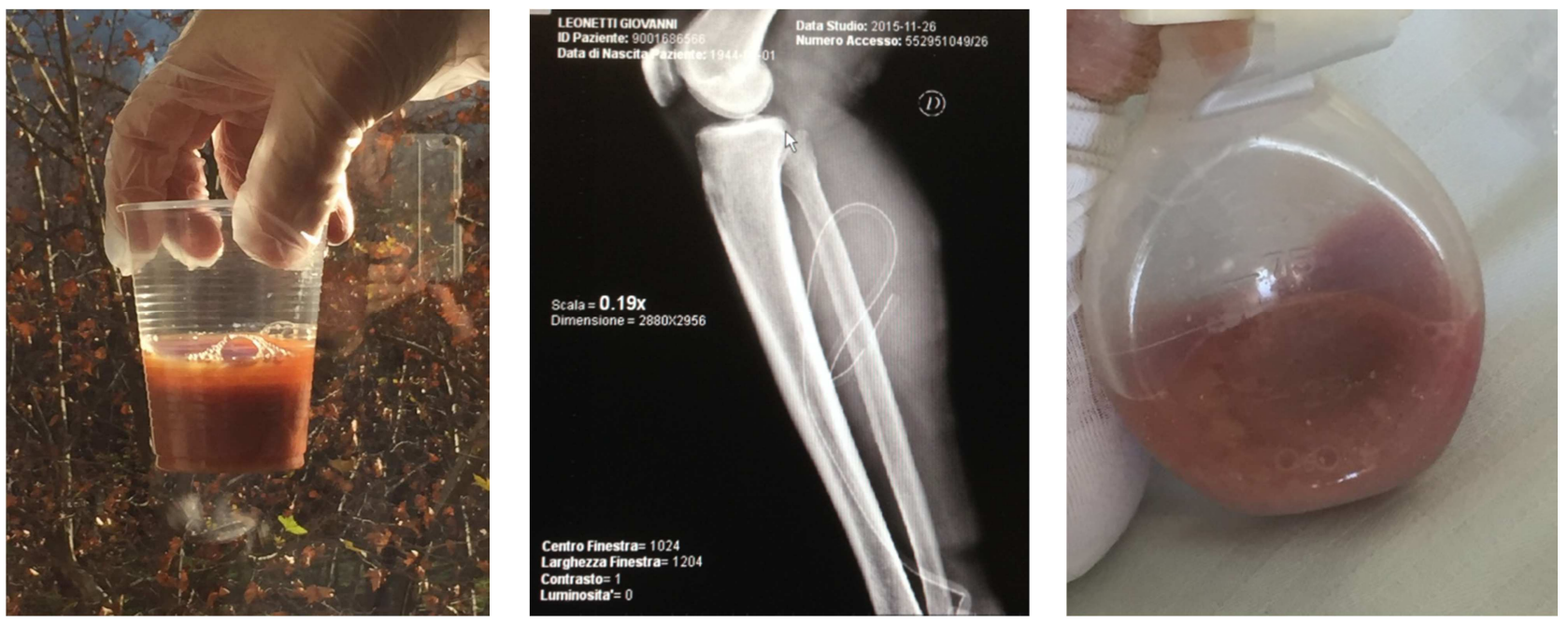